\title{
Exciton spin coherence in InGaAs/ GaAs quantum dots revisited by heterodyne pump-probe experiment (Withdrawal Notice)
}

B. Eble, B. Siarry, F. Bernardot, P. Grinberg, C. Testelin, et al.

B. Eble, B. Siarry, F. Bernardot, P. Grinberg, C. Testelin, A. Lemaître, "Exciton spin coherence in InGaAs/GaAs quantum dots revisited by heterodyne pumpprobe experiment (Withdrawal Notice)," Proc. SPIE 9931, Spintronics IX, 993121 (6 December 2016); doi: 10.1117/12.2236319

SPIE Event: SPIE Nanoscience + Engineering, 2016, San Diego, California, United States 


\title{
Exciton spin coherence in InGaAs/GaAs quantum dots revisited by heterodyne pump-probe experiment (Withdrawal Notice)
}

\author{
Proc. SPIE 9931, $993121(2016)$ \\ Online Publication Date: 11 November 2016 \\ Withdrawn from Publication: 1 December 2016 \\ Conference Date: 8 August-1 September 2016 \\ Conference Location: San Diego, California, United States \\ Conference Title: Spintronics IX \\ Conference Chairs: Henri-Jean Drouhin, Jean-Eric Wegrowe, Manijeh Razeghi \\ B. Eble, B. Siarry, F. Bernardot, P. Grinberg and C. Testelin \\ Sorbonnes Univ., Univ. Paris 6, CNRS, Institut des NanoSciences de Paris \\ A. Lemaitre \\ CNRS, Lab. de Photonique et de Nanostructures (France)
}

This paper has been withdrawn at the request of the authors. 